\title{
Magnetic phenomena in Co-containing layered double hydroxides
}

Daniel E. L. Vieira, Andrei N. Salak, Alexey V. Fedorchenko, Yurii G. Pashkevich, Elena L. Fertman, Vladimir A.

Desnenko, Roman Yu. Babkin, Erik Čižmár, Alexander Feher, Augusto B. Lopes, and Mário G. S. Ferreira

Citation: Low Temperature Physics 43, 977 (2017); doi: 10.1063/1.5001299

View online: http://dx.doi.org/10.1063/1.5001299

View Table of Contents: http://aip.scitation.org/toc/ltp/43/8

Published by the American Institute of Physics

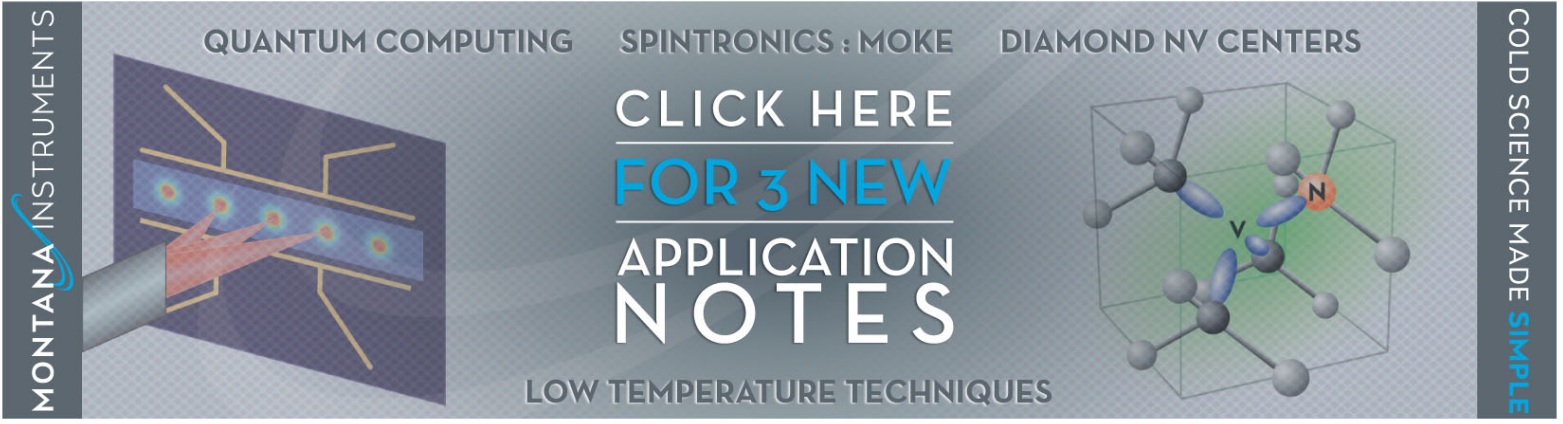




\title{
Magnetic phenomena in Co-containing layered double hydroxides
}

\author{
Daniel E. L. Vieira and Andrei N. Salak ${ }^{a)}$
}

Department of Materials and Ceramic Engineering, CICECO - Aveiro Institute of Materials, University of Aveiro, 3810-193 Aveiro, Portugal

\begin{abstract}
Alexey V. Fedorchenko
Institute of Physics, Faculty of Science, P.J. Šafárik University in Košice 9 Park Angelinum, Košice 04154, Slovakia and B. Verkin Institute for Low Temperature Physics and Engineering, National Academy of Sciences of Ukraine, 47 Nauky Ave., Kharkiv 61103, Ukraine
\end{abstract}

\section{Yurii G. Pashkevich}

B. Verkin Institute for Low Temperature Physics and Engineering, National Academy of Sciences of Ukraine, 47 Nauky Ave., Kharkiv 61103, Ukraine and O. Galkin Donetsk Institute for Physics and Engineering, National Academy of Sciences of Ukraine, 46 Nauky Ave., Kyiv 03680, Ukraine

\section{Elena L. Fertman and Vladimir A. Desnenko}

B. Verkin Institute for Low Temperature Physics and Engineering, National Academy of Sciences of Ukraine, 47 Nauky Ave., Kharkiv 61103, Ukraine

\section{Roman Yu. Babkin}

O. Galkin Donetsk Institute for Physics and Engineering, National Academy of Sciences of Ukraine, 46 Nauky Ave., Kyiv 03680, Ukraine

\section{Erik Čižmár and Alexander Feher}

Institute of Physics, Faculty of Science, P.J. Šafárik University in Košice 9 Park Angelinum, Košice 04154, Slovakia

Augusto B. Lopes and Mário G. S. Ferreira

Department of Materials and Ceramic Engineering, CICECO - Aveiro Institute of Materials, University of Aveiro, 3810-193 Aveiro, Portugal

(Submitted February 17, 2017)

Fiz. Nizk. Temp. 43, 1214-1218 (August 2017)

Magnetic behavior of $\mathrm{Co}^{\mathrm{II}}(n) \mathrm{Al}^{\mathrm{III}}$ layered double hydroxides (LDHs) $(n=\mathrm{Co} / \mathrm{Al}=2$ and 3 ) intercalated with nitrate was studied as a function of temperature. Both LDH compounds are paramagnetic above about $8 \mathrm{~K}$. A rapid increase of their magnetic moments occurs below this temperature until the moments reach the maximum values at $T_{\max }$ of $4.0 \mathrm{~K}$ and $3.2 \mathrm{~K}$ for $\mathrm{Co}(2) \mathrm{Al}-\mathrm{NO}_{3}$ and $\mathrm{Co}(3) \mathrm{Al}-\mathrm{NO}_{3}$, respectively. Below $T_{\max }$, the zero-field-cooled and the field-cooled static magnetization curves are strongly different. Along with this low-temperature phenomena, $\mathrm{Co}(2) \mathrm{Al}-\mathrm{NO}_{3}$ and $\mathrm{Co}(3) \mathrm{Al}-\mathrm{NO}_{3}$ demonstrate anomalous behavior of their temperature dependence magnetic susceptibility in a highertemperature range: between 75 and $175 \mathrm{~K}$, both the paramagnetic Curie temperature and the effective magnetic moment change in a non-monotonous way. Possible structural reasons of the observed magnetic behavior of the $\mathrm{Co}^{\mathrm{II}}(n) \mathrm{Al}^{\mathrm{III}}$ LDHs are discussed. Published by AIP Publishing.

[http://dx.doi.org/10.1063/1.5001299]

\section{Introduction}

Layered double hydroxides (LDHs) are composed of positively-charged mixed metal $\mathrm{M}^{\mathrm{II}}-\mathrm{M}^{\mathrm{III}}$ hydroxide layers with change-compensating anions $\mathrm{A}^{y}$ - and water molecules intercalated into interlayers. ${ }^{1}$ The metal cations in the layers are coordinated by six oxygen atoms forming $2 \mathrm{D}$ structures of edge-linked oxygen octahedra. General formula of the most common LDHs can be represented as $\left[\mathrm{M}_{1-x}^{\mathrm{I}} \mathrm{M}_{x}^{\mathrm{III}}(\mathrm{OH})_{2}\right]^{x+}\left(\mathrm{A}^{y-}\right)_{x / y} \cdot z \mathrm{H}_{2} \mathrm{O}$. The parameters $x$ and $z$ can vary over the wide ranges thereby enable a flexibility of LDH structure allowing intercalation of anions with different size and charge. ${ }^{2-4}$
LDHs are typically characterized by hexagonal symmetry with the $c$-axis perpendicular to the layers (space group $R \overline{3} \mathrm{~m}$ ). The $a$ parameter is a function of both size and ratio of cations $\mathrm{M}^{\mathrm{II}}$ and $\mathrm{M}^{\mathrm{III}}$.,6 The $c$ parameter depends mainly on size, charge and orientation of the inter-calated species. $5,7,8$

The main applications of LDHs are anion exchange and catalysis. At the same time, their layered structure in combination with a controllable interlayer distance offer a unique opportunity to induce and study magnetic phenomena in these $2 \mathrm{D}$ materials.

LDHs containing either $\mathrm{Ni}^{\mathrm{II}}$ or $\mathrm{Co}^{\mathrm{II}}$ as a divalent cation were prepared and studied in respect of magnetic properties. ${ }^{9-13}$ 
Onset of spontaneous magnetization and a peak of magnetic susceptibility were observed in those compounds at low temperatures. However, no long-range magnetic ordering was revealed and those "magnetic LDHs" were considered as spin glasses. It was found that in $\mathrm{Ni}^{\mathrm{II}}$-containing $\mathrm{LDHs}$, the magnetic susceptibility peak is at higher temperature than the respective peak in $\mathrm{Co}^{\mathrm{II}}$-containing LDHs. At the same time, for an $\mathrm{LDH}$ with a certain diva-lent cation (either cobalt or nickel), the onset temperature for the spontaneous magnetization was shown to depend on the interlayer gallery height rather than on nature $\left(\mathrm{Fe}^{\mathrm{III}}, \mathrm{Cr}^{\mathrm{III}}, \mathrm{Mn}^{\mathrm{III}}\right.$ or $\left.\mathrm{Al}^{\mathrm{III}}\right)$ of the trivalent cation and the $\mathrm{M}^{\mathrm{II}}$ to $\mathrm{M}^{\mathrm{III}}$ ratio. Moreover, intercalation of those LDHs with a magnetic anionic complex instead of a non-magnetic complex of the same size has a negligible effect on the magnetic properties. ${ }^{12}$ It should be noticed that nickel-containing LDHs have been studied in much more detail than cobalt-containing ones.

In this work, we report on the preliminary results of a systematic study of the layered double hydroxides of the $\left[\mathrm{Co}_{1-x}^{\mathrm{II}} \mathrm{Al}_{x}^{\mathrm{III}}(\mathrm{OH})_{2}\right]^{x+}\left(\mathrm{A}^{y-}\right)_{x / y} \cdot z \mathrm{H}_{2} \mathrm{O}$ system (hereafter $\mathrm{Co}(n)$ Al-A, where $n=(1-x) / x$ is the $\mathrm{Co} / \mathrm{Al}$ molar ratio). Magnetic properties of two compositions of the system with $n=2$ and 3 , and $\mathrm{A}=\mathrm{NO}_{3}^{-}$are compared and analyzed based on both experimental data and theoretical calculations.

\section{Experimental}

$\mathrm{Co}(n) \mathrm{Al}-\mathrm{NO}_{3}$ LDHs with $n=2$ and 3 were synthesized using the conventional co-precipitation method based on the experimental procedure applied to prepare $\mathrm{Mg}(2) \mathrm{Al}-\mathrm{NO}_{3}$ and $\mathrm{Zn}(2) \mathrm{Al}-\mathrm{NO}_{3} .{ }^{4}$ Cobalt (II) nitrate hexahydrate $(\geq 98 \%)$, aluminum nitrate nonahydrate $(\geq 98.5 \%)$, sodium hydroxide $(\geq 98 \%)$, and sodium nitrate $(\geq 99.5 \%)$ served as the reagents. All the solutions were prepared in distilled deaerated water. Proportions of the reagents in the solutions were to meet either the $2: 1$ or $3: 1$ ratio of $\mathrm{Co}$ and $\mathrm{Al}$. The coprecipitation was carried out at $\mathrm{pH} 8$. In order to complete crystallization, the obtained suspension was kept in a water bath for $24 \mathrm{~h}$ followed by centrifugation. The resulting dense slurry was washed with deaerated water and centrifuged again. The final product was dried at $330 \mathrm{~K}$ for $24 \mathrm{~h}$.

X-ray diffraction (XRD) study of the obtained LDH powders was performed using a Rigaku D/MAX-B diffractometer equipped with a graphite monochromator to reduce contributions to the XRD data caused by fluorescence of cobalt in $\mathrm{Cu} \mathrm{K}_{\alpha}$ radiation. XRD data were collected in a step mode (step $0.02^{\circ}, 20 \mathrm{~s}$ per step) at room temperature.

Size and morphology of the LDH particles/crystallites were characterized using a Hitachi S-4100 scanning electron microscope (SEM) and a Hitachi 9100 transmission electron microscope (TEM) with an electron beam acele-rating voltages of 25 and $300 \mathrm{kV}$, respectively.

For magnetic measurements, the LDH powders were compacted into discs of about $3 \mathrm{~mm}$ in diameter and about $0.3 \mathrm{~mm}$ thick. Static magnetic properties of these samples were studied using a SQUID magnetometer technique (Quantum Design MPMS-3) between 2 and $300 \mathrm{~K}$ in the magnetic field of 100 Oe under both zero-field-cooled (ZFC) and field-cooled (FC) conditions.

\section{Results and discussion}

\subsection{Structural and morphological characterization}

XRD patterns of the obtained materials are shown in Fig. 1. The diffraction reflections typical of $\mathrm{LDH}$ structure are clearly seen. No reflections that can be attributed to other phase(s) have been observed. The patterns are indexed in the $3 \mathrm{R}$ polytype ${ }^{1}$ for which $c=3 d$ where $d$ is the basal spacing (the distance between the adjacent $\mathrm{M}^{\mathrm{II}}-\mathrm{M}^{\mathrm{III}}$ hydroxide layers). The basal spacing can be represented as a sum of the layer thickness and the interlayer gallery height.

The lattice parameters of the LDHs were calculated from the angle positions of the diffraction reflections (110), (003) and (006) to be $a=3.064 \AA, c=26.729 \AA$, and $a=3.067 \AA$, $c=26.670 \AA$ for $\mathrm{Co}(2) \mathrm{Al}-\mathrm{NO}_{3}$ and $\mathrm{Co}(3) \mathrm{Al}-\mathrm{NO}_{3}$, respectively. Since the ionic radius of $\mathrm{Co}^{\mathrm{II}}$ is bigger than that of $\mathrm{Al}^{\mathrm{III}}$ in the same octahedral coordination, the $\mathrm{LDH}$ compound with a higher $\mathrm{Co} / \mathrm{Al}$ ratio has a higher $a$ parameter value. Because of this cation size difference, one can expect that the mixed metal hydroxide layer in $\mathrm{Co}(3) \mathrm{Al}-\mathrm{NO}_{3}$ is thicker than that in $\mathrm{Co}(2) \mathrm{Al}-\mathrm{NO}_{3}$. However, an increase of the divalentto-trivalent cation ratio results in a decrease of the layer charge, $+x$ (see the general formula of $\mathrm{M}^{\mathrm{II}}-\mathrm{M}^{\mathrm{III}} \mathrm{LDH}$ in Introduction). Hence, nitrate anions which are tilted with some slant angle (against the layer plane) in the interlayers to compensate the layer charge ${ }^{7}$ can be arranged with a lower angle. It implies a smaller interlayer gallery height and, thereby, a lower $c$ parameter value.

As seen from Fig. 1, the diffraction peaks of the obtained LDHs are rather wide but without evident asymmetry. The latter indicates that the observed peak broadening is likely to be caused by small crystallite size of the materials. Indeed, it was found from SEM and TEM observations that,

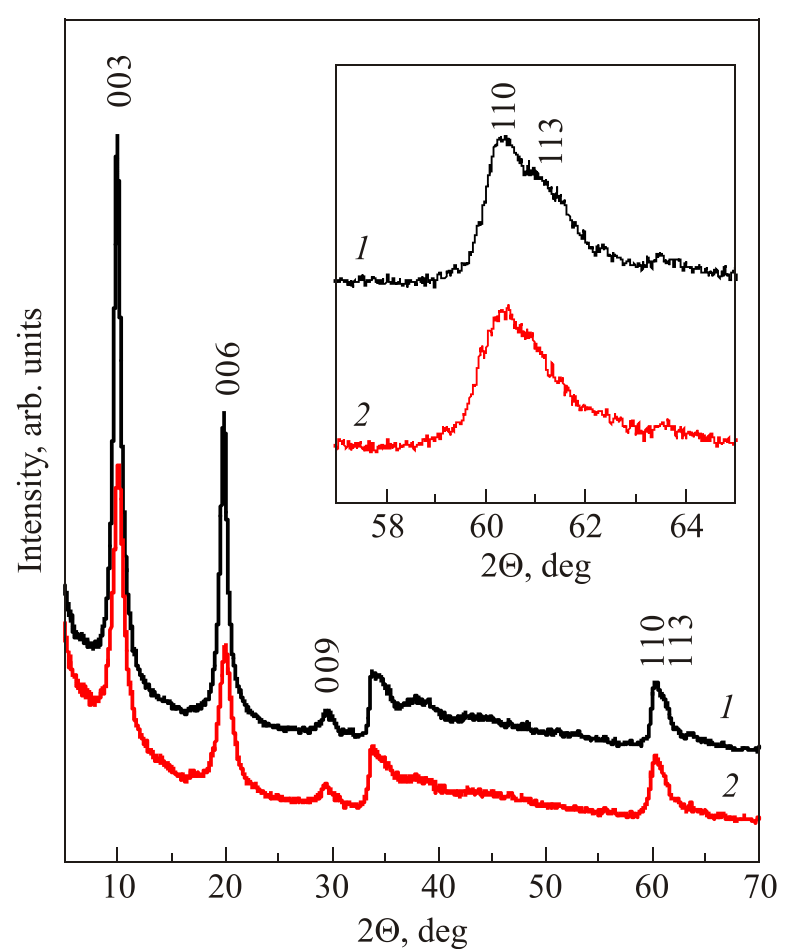

Fig. 1. XRD patterns of (a) $\mathrm{Co}(2) \mathrm{Al}-\mathrm{NO}_{3}$ (l) and (b) $\mathrm{Co}(3) \mathrm{Al}-\mathrm{NO}_{3}$ (2) compounds. The most characteristic diffraction reflections of the $\mathrm{LDH}$ phases are indexed. Inset shows the patterns in the range of (110) and (113) reflections. 
although the average size of the LDH particles is close to 1 $\mu \mathrm{m}$, these particles are actually agglomerates of the flakelike hexagonal crystallites [Fig. 2(a)]. The average crystallite size was estimated to be about $100 \mathrm{~nm}$ for $\mathrm{Co}(2) \mathrm{Al}-\mathrm{NO}_{3}$ [Fig. 2(b)] and about $50 \mathrm{~nm}$ for $\mathrm{Co}(3) \mathrm{Al}-\mathrm{NO}_{3}$.

\subsection{Magnetic properties}

$\mathrm{ZFC}$ and $\mathrm{FC}$ static magnetizations of $\mathrm{Co}(2) \mathrm{Al}-\mathrm{NO}_{3}$ and $\mathrm{Co}(3) \mathrm{Al}-\mathrm{NO}_{3} \mathrm{LDHs}$ as a function of temperature measureed in the magnetic field of 100 Oe are shown in Fig. 3. For each compound, a rapid increase of the magnetic moment is seen below about $8 \mathrm{~K}$ until it reaches the maximum value $M_{\max }$ at the temperature $T_{\max }$. Both $T_{\max }$ and $M_{\max }$ are dependent on the sample composition. $T_{\max }$ is 4.0 and $3.2 \mathrm{~K}$, while $M_{\max }$ is about $1130 \mathrm{emu} / \mathrm{mol}$ and $790 \mathrm{emu} / \mathrm{mol}$, for $\mathrm{Co}(2) \mathrm{Al}-\mathrm{NO}_{3}$ and $\mathrm{Co}(3) \mathrm{Al}-\mathrm{NO}_{3}$, respectively. As the onset of the magnetic moment has to be a function of both the interlayer distance and the cation ratio, the observed differences in values of $T_{\text {max }}$ and $M_{\text {max }}$ for $\mathrm{Co}(2) \mathrm{Al}-\mathrm{NO}_{3}$ and $\mathrm{Co}(3) \mathrm{Al}-\mathrm{NO}_{3}$ may be associated with a complex interplay between in-layer and intra-layer exchange. Besides, it is known that size effects are very strong in nano-dimensional structures. ${ }^{14}$ In this respect, one can assume that the magnetic properties of $\mathrm{Co}(3) \mathrm{Al}-\mathrm{NO}_{3}$ are affected stronger than those of $\mathrm{Co}(2) \mathrm{Al}-\mathrm{NO}_{3}$ since the former has a twice smaller average crystallite size than the latter $(50 \mathrm{~nm}$ against $100 \mathrm{~nm})$.

Below $T_{\max }$, the ZFC and the FC magnetization curves measured in low magnetic field are strongly different which may be associated with either the anisotropy of the compounds or their magnetic glass behavior.

Unusual behavior of the inverse magnetic susceptibility has been found in both studied LDH compounds in the range
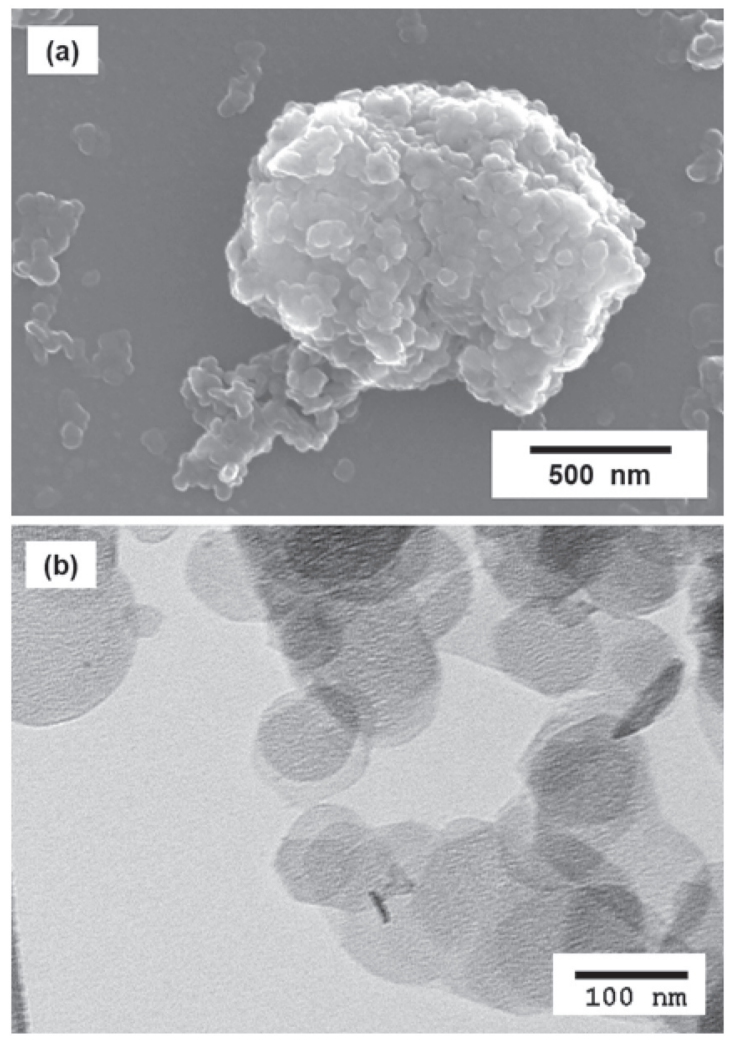

Fig. 2. (a) SEM image of a particle of $\mathrm{Co}(2) \mathrm{Al}-\mathrm{NO}_{3} \mathrm{LDH}$. (b) TEM image of the dispersed crystallites of $\mathrm{Co}(2) \mathrm{Al}-\mathrm{NO}_{3} \mathrm{LDH}$.

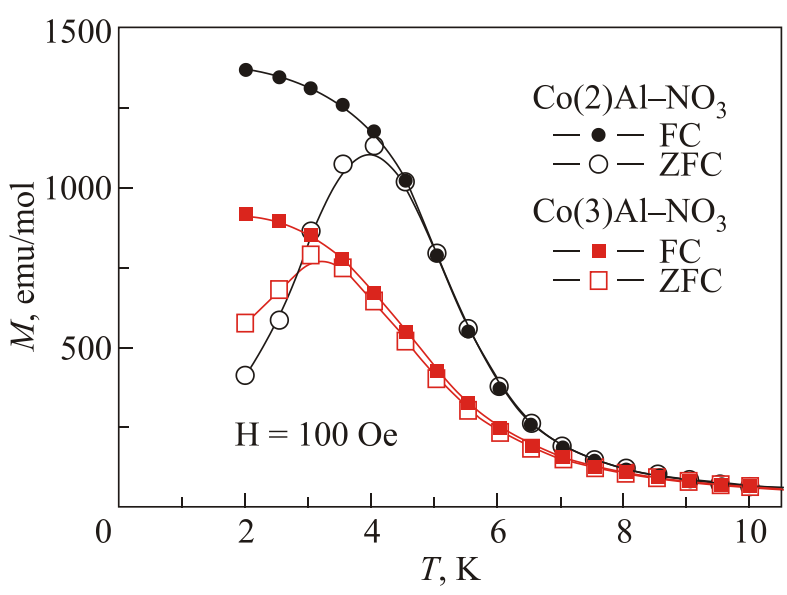

Fig. 3. ZFC and FC static magnetizations of $\mathrm{Co}(2) \mathrm{Al}-\mathrm{NO}_{3}$ and $\mathrm{Co}(3) \mathrm{Al}-\mathrm{NO}_{3}$ measured in the magnetic field of $100 \mathrm{Oe}$ as a function of temperature.

of $75-175 \mathrm{~K}$. Both $\mathrm{Co}(2) \mathrm{Al}-\mathrm{NO}_{3}$ and $\mathrm{Co}(3) \mathrm{Al}-\mathrm{NO}_{3}$ are paramagnetic above about $8 \mathrm{~K}$. However, for each compound, the temperature dependent inverse magnetic susceptibility, $1 / \chi(T)$, in paramagnetic state cannot be well approximated by a single Curie-Weiss line. The best fit has been achieved when a higher-temperature range and a lowertemperature range were approximated by different lines (Fig. 4 , Table 1). The obtained positive values of the paramagnetic Curie temperature $(\Theta)$ in both ranges imply a ferromagnetic character of the interaction between cobalt ions in both $\mathrm{Co}(2) \mathrm{Al}-\mathrm{NO}_{3}$ and $\mathrm{Co}(3) \mathrm{Al}-\mathrm{NO}_{3}$. It should be noticed that the $\Theta$ values corresponding to the lower- $T$ range are smaller than those corresponding to the higher- $T$ range (Table 1) which suggests reducing of the ferromagnetic correlations between cobalt ions with decreasing temperature. The anomaly found is clearly seen in the temperature dependence of the effective magnetic moment, $\mu_{\text {eff }}(T)$ (Fig. 5). It may be caused by structural reasons.

Let us briefly discuss the possible structural origin of the unexpected temperature variations of $\Theta$ and $\mu_{\text {eff }}$ observed in both LDH compounds under study. The low lying energy levels of the Kramers $\mathrm{Co}^{\mathrm{II}}$ ions $\left(3 d^{7}\right.$-configuration) are

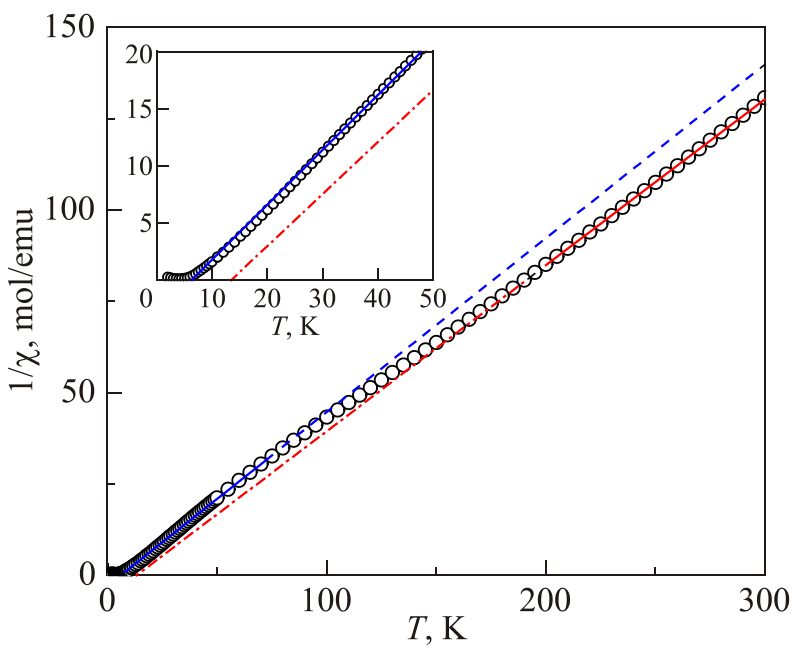

Fig. 4. Temperature dependence inverse magnetic susceptibility, $1 / \chi(T)$, of $\mathrm{Co}(2) \mathrm{Al}-\mathrm{NO}_{3}$ measured in magnetic field of $100 \mathrm{Oe}$. The lines are the Curie-Weiss approximations, $\chi=C / T-\Theta$, in the lower-temperature and the higher-temperature paramagnetic ranges. Inset: Magnification of the lowertemperature range. 
TABLE 1 . The parameters obtained by the Curie-Weiss fitting of the temperature dependent magnetic susceptibility of the LDH compounds.

\begin{tabular}{lccc}
\hline \hline Composition & Temperature range $(\mathrm{K})$ & $C(\mathrm{emu} \cdot \mathrm{K} / \mathrm{mol})$ & $\Theta(\mathrm{K})$ \\
\hline $\mathrm{Co}(2) \mathrm{Al}-\mathrm{NO}_{3}$ & $8-75$ & 2.1 & 6.2 \\
& $200-300$ & 2.2 & 13.5 \\
$\mathrm{Co}(3) \mathrm{Al}-\mathrm{NO}_{3}$ & $8-85$ & 2.1 & 7.4 \\
& $175-300$ & 2.2 & 12.5 \\
\hline \hline
\end{tabular}

responsible for magnetic properties of these LDHs. In an averaged LDH structure description, a virtual $\mathrm{M}_{1-x}^{\mathrm{II}} \mathrm{M}_{x}^{\mathrm{III}}$ cation occupies the center of oxygen octahedron with the $\mathrm{D}_{3 \mathrm{~d}}$ symmetry. ${ }^{15}$ In such an atomic coordination, combination of the crystal field with the spin-orbit interaction leads to the splitting of the ground ${ }^{4} \mathrm{~F}$ term of the free $\mathrm{Co}^{\mathrm{II}}$ ion. As a result, the four lowest Kramers doublets have the energies which became actual for magnetic properties below room temperature. ${ }^{16,17}$ All the Kramers doublets are characterized by the highly anisotropic $g$-tensors caused by the $\mathrm{C}_{3}$ axial symmetry.

The following two distinctive features of the LDH compounds under study make their $\mathrm{Co}^{\mathrm{II}}$ ions spectra different from the standard expectation. First, the octahedral cage is not actually formed by $\mathrm{O}^{2-}$ ions but by $\mathrm{OH}^{-}$ones which considerably suppress the crystal field strength. Second, the Co ${ }^{\text {II }}$ octahedral cages are distorted and have symmetry lower than $\mathrm{D}_{3 \mathrm{~d}}$ since their nearest-neighbor sites are occupied by $\mathrm{Al}^{\mathrm{III}}$ with a notably smaller ionic radius. Because of a weak crystal field, the $\mathrm{Co}^{\mathrm{II}}$ ions remain in the high spin state $(S=3 / 2)$.

Our preliminary theoretical calculations demonstrate some convergence in energies of the two lowest Kramers doublets due to the first feature and, therefore, a high sensitivity to the octahedral cage distortions associated with the second feature. Furthermore, the $g$-factor values of both doublets are very sensitive to the small deviation of the $\mathrm{OH}-$ bond direction from the 3-fold axis perpendicular to the mixed metal hydroxide layers.

Based on this observation one can propose the following explanation of the anomalous temperature variations of $\Theta$

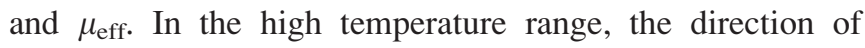
hydroxyl groups oscillates in a way that its average direction

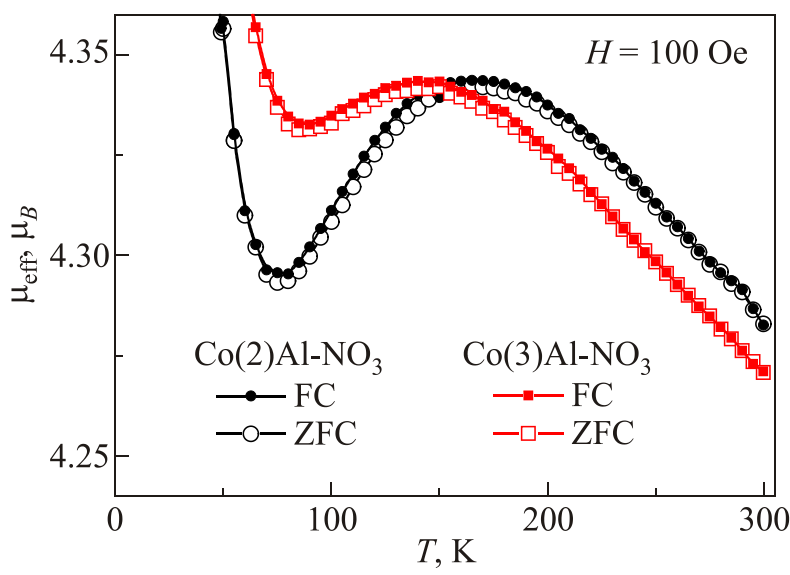

Fig. 5. The effective magnetic moment, $\mu_{\text {eff }}$, calculated from the temperature dependence magnetic susceptibility of $\mathrm{Co}(2) \mathrm{Al}-\mathrm{NO}_{3}$ and $\mathrm{Co}(3) \mathrm{Al}-\mathrm{NO}_{3}$ (measured in magnetic field of 100 Oe in the temperature range of $8-300 \mathrm{~K}$ ) using the equation $\mu_{\text {eff }}=\sqrt{3 k_{B} C /\left(N_{A} \mu_{B}^{2}\right)}$, where $k_{B}$ is a Boltzmann constant, $N_{A}$ is an Avogadro number, $\mu_{B}$ is a Bohr magneton, and $C$ is the Curie constant. is parallel to the $C_{3}$ axis. Therefore, all the $\mathrm{OH}^{-}$groups participate in the interlayer superexchange bonding. The intercalated anions are also expected to be involved in the interlayer magnetic exchange paths. According to the chemical formula of the LDHs under study, $\left[\mathrm{Co}_{1-x}^{\mathrm{II}} \mathrm{Al}_{x}^{\mathrm{III}}(\mathrm{OH})_{2}\right]^{x+}$ $\left(\mathrm{A}^{y-}\right)_{x / y} \cdot z \mathrm{H}_{2} \mathrm{O}$, the molar content of the intercalated anions per formula unit, $x / y$, is dependent on the $\mathrm{Co} / \mathrm{Al}$ cation ratio. Hence, the relative amount of the anions involved in the exchange path is bigger in $\mathrm{Co}(2) \mathrm{Al}-\mathrm{NO}_{3}$ than in $\mathrm{Co}(3) \mathrm{Al}-\mathrm{NO}_{3}$. As a result, for temperatures above $200 \mathrm{~K}$, the Curie temperature value for the former is higher than that for the latter. It should be mentioned that both the intraand the interlayer exchanges contribute to the Curie temperature value. As temperature decreases, orientation of the hydroxyl groups become frozen and a part of them are tilted in accordance with the Al-caused corrugation of the octahedra layer surface. The tilted hydro-xyl groups weaken the interlayer magnetic exchange paths. Therefore, the averaged Curie temperature is expected to be lower for the LDH compound with a higher Al content (per formula unit) that is in agreement with the experimental data (see Table 1). The proposed scenario also explains the observed non-monotonic temperature behavior of the effective magnetic moment, since $\mu_{\text {eff }}$ includes an average value of the $\mathrm{Co}^{\mathrm{II}} g$-factors which are sensitive to orientation of the hydroxyl groups.

Hydroxyl groups are the main structural units of the layered double hydroxides. Therefore the observed magnetic anomalies should be characteristic of all the $\left[\mathrm{M}_{1-x}^{\mathrm{I}} \mathrm{M}_{x}^{\mathrm{III}}(\mathrm{OH})_{2}\right]^{x+}$ $\left(\mathrm{A}^{y-}\right)_{x / y} \cdot z \mathrm{H}_{2} \mathrm{O}$ compounds with notably different radii of the magnetic $\mathrm{M}^{\mathrm{II}}$ and the non-magnetic $\mathrm{M}^{\mathrm{III}}$ cations.

\section{Conclusions}

We have studied the static magnetic properties of the $\mathrm{Co}(2) \mathrm{Al}-\mathrm{NO}_{3}$ and $\mathrm{Co}(3) \mathrm{Al}-\mathrm{NO}_{3}$ layered double hydroxides (LDHs) over a temperature range of $2-300 \mathrm{~K}$. Both LDHs are paramagnetic above $8 \mathrm{~K}$. For each compound, a rapid increase of the magnetic moment is observed below this temperature until it reaches the maximum value at $T_{\max }$. Below $T_{\max }$, which are $4.0 \mathrm{~K}$ and $3.2 \mathrm{~K}$ for $\mathrm{Co}(2) \mathrm{Al}-\mathrm{NO}_{3}$ and $\mathrm{Co}(3) \mathrm{Al}-\mathrm{NO}_{3}$, respectively, the temperature dependences of the zero-field-cooled and the field-cooled magnetizations are essentially different that suggests either the anisotropy of the compounds or their magnetic glass behavior.

In both $\mathrm{Co}(2) \mathrm{Al}-\mathrm{NO}_{3}$ and $\mathrm{Co}(3) \mathrm{Al}-\mathrm{NO}_{3}$, an anomalous temperature behavior of the inverse magnetic susceptibility was detected between about 75 and $175 \mathrm{~K}$. The Curie- Weiss linear approximation of the observed temperature dependent inverse magnetic susceptibility is different in the paramagnetic ranges above and below the anomaly. The paramagnetic Curie temperature $(\Theta)$ values obtained from the highertemperature range data are about twice higher than those obtained from the lower-temperature range ones which suggests reducing of the ferromagnetic correlations between cobalt ions with decreasing temperature. Besides, in the range of $75-175 \mathrm{~K}$, the effective magnetic moment $\left(\mu_{\text {eff }}\right)$ calculated from the temperature dependent magnetic susceptibility of both LDH compounds varies in a non-monotonic way.

The observed anomalies of $\Theta$ and $\mu_{\text {eff }}$ can be theoretically explained in terms of temperature dependent orientation of the hydroxyl groups and distortions of the $\mathrm{Co}^{\mathrm{II}}$-containing 
octahedra in the mixed metal hydroxide layers of LDHs. In order to clarify the origin of the anomaly, a particular lowtemperature structural study is required.

This paper is dedicated to Professor Viktor Valentinovich Eremenko on the occasion of his 85th anniversary. A. Feher, Y. Pashkevich, E. Fertman, V. Desnenko, A. Fedorchenko, are very thankful to Professor Eremenko for decades of fruitful collaboration. Viktor Valentinovich was and remains an excellent mentor in Magnetism for the whole Košice Low Temperature Group.

The work was supported by project TUMOCS. This project has received funding from the European Union's Horizon 2020 research and innovation program under the Marie Skłodowska-Curie grant Agreement No. 645660. The financial supports of the Slovak Research and Development Agency under Contract Nos. APVV-14-0073 and APVV0132-11, and the supports of P2020 COMPETE and FCTPortugal through project POCI-01-0145-FEDER-016686PTDC/CTM-NAN/2418/2014 (NANOCONCOR) are gratefully acknowledged as well.

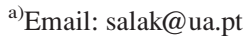

${ }^{1}$ D. E. Evans and R. C. T. Slade, Structure \& Bonding (Springer-Verlag, Berlin, Germany, 2005), Vol. 119, p. 1.
${ }^{2}$ A. I. Khan and D. O'Hare, J. Mater. Chem. 12, 3191 (2002).

${ }^{3}$ A. N. Salak, J. Tedim, A. I. Kuznetsova, L. G. Vieira, J. L. Ribeiro, M. L. Zheludkevich, and M. G. S. Ferreira, J. Phys. Chem. C 117, 4152 (2013).

${ }^{4}$ M. Serdechnova, A. N. Salak, F. S. Barbosa, D. E. L. Vieira, J. Tedim, M.

L. Zheludkevich, and M. G. S. Ferreira, J. Solid State Chem. 233, 158 (2016).

${ }^{5}$ S. P. Newman and W. Jones, Supramolecular Organization and Materials Design (Cambridge University Press, Cambridge, 2001), p. 295.

${ }^{6}$ I. G. Richardson, Acta Cryst. B 69, 414 (2013).

${ }^{7}$ A. N. Salak, J. Tedim, A. I. Kuznetsova, J. L. Ribeiro, L. G. Vieira, M. L. Zheludkevich, and M. G. S. Ferreira, Chem. Phys. 397, 102 (2012).

${ }^{8}$ A. N. Salak, A. D. Lisenkov, M. L. Zheludkevich, and M. G. S. Ferreira, ECS Electrochem. Lett. 3, 9 (2014).

${ }^{9}$ M. Intissar, R. Segni, C. Payen, J. P. Besse, and F. Leroux, J. Solid State Chem. 167, 508 (2002).

${ }^{10}$ E. Coronado, J. R. Galan-Mascaros, C. Marti-Gastaldo, A. Ribera, E. Palacios, M. Castro, and R. Burriel, Inorg. Chem. 47, 9103 (2008).

${ }^{11}$ J. J. Almansa, E. Coronado, C. Martí-Gastaldo, and A. Ribera, Eur. J. Inorg. Chem. 36, 5642 (2008).

${ }^{12}$ E. Coronado, C. Martí-Gastaldo, E. Navarro-Moratalla, and A. Ribera, Appl. Clay Sci. 48, 228 (2010).

${ }^{13}$ F. Giovannelli, M. Zaghrioui, C. Autret-Lambert, F. Delorme, A. Seron, T. Chartier, and B. Pignon, Mater. Chem. Phys. 137, 55 (2012).

${ }^{14}$ J. M. Wesselinowa, J. Magn. Magn. Mater. 322, 234 (2010).

${ }^{15}$ I. G. Richardson, Acta Cryst. B 69, 150 (2013).

${ }^{16}$ V. F. Meshcheryakov, J. Exp. Theor. Phys. 98, 144 (2004).

${ }^{17}$ R. Babkin, K. V. Lamonova, S. M. Orel, Yu. G. Pashkevich, and V. F. Meshcheryakov, Opt. Spectrosc. 112, 438 (2012).

This article was published in English in the original Russian journal. Reproduced here with stylistic changes by AIP Publishing. 